\title{
Desigualdades sociais e alimentação complementar na América Latina e no Caribe
}

\author{
Social inequalities and complementary feeding in Latin America \\ and the Caribbean
}

Allyevison Ulisses Alves Cavalcanti (https://orcid.org/0000-0001-7153-8578) ${ }^{1}$

Cristiano Siqueira Boccolini (https://orcid.org/0000-0002-4804-5641) ${ }^{2}$
${ }^{1}$ Escola Nacional de Saúde Pública Sergio Arouca, Fundação Oswaldo Cruz. R. Leopoldo Bulhões 1480, Manguinhos. 21041-210 Rio de Janeiro RJ Brasil. auacavalcanti@gmail.com ${ }^{2}$ Instituto de Comunicação e Informação Científica e Tecnológica em Saúde, Fundação Oswaldo Cruz. Rio de Janeiro RJ Brasil.

\begin{abstract}
This study evaluated the role of social inequalities in complementary feeding patterns between and within countries in Latin America and the Caribbean. This ecological study employed aggregate data from population-based surveys. The units of analysis were all 16 countries in Latin America and the Caribbean for which information was available in the Demographic and Health Surveys (DHS) and Multiple Indicator Cluster Surveys (MICS) databases. The outcomes selected were the prevalences indicated by the "introduction of solid, semi-solid or soft foods" (ISS$S F)$, "minimum dietary diversity" (MDD), " $m i$ nimum meal frequency" (MMF) and "minimum acceptable diet" (MAD) standardised complementary feeding indicators recommended by the WHO and available in UNICEF databases. The differences in prevalences between the wealthiest and poorest income quintiles were calculated in absolute and relative terms. Comparing the four indicators of complementary feeding, the ISSSF showed best performance, returning prevalence above $80 \%$ in 10 of the 11 countries evaluated. The indicator showing worst performance was the $M A D$, with prevalence above $60 \%$ in only one of the eight countries evaluated. In almost all countries, by all indicators, prevalences of complementary feeding were lower in the poorest population groups than in the wealthiest.
\end{abstract}

Key words Complementary feeding, Social determinants of health, Public health, Nutrition policy
Resumo Este artigo tem como objetivo avaliar o papel das desigualdades sociais nos padrões de alimentação complementar nos países da América Latina e do Caribe. Trata-se de um estudo ecológico que utilizou dados agregados de inquéritos de base populacional. A unidade de análise da pesquisa foram 16 países da América Latina $e$ do Caribe com informações disponíveis. Foram selecionados como desfechos a prevalência dos seguintes indicadores de alimentação complementar: introdução alimentar de alimentos sólidos, semissólidos e pastosos" (IASSP), "diversidade alimentar mínima" (DAM), "frequência alimentar mínima" (FAM) e "alimentação mínima aceitável” (AMA). As diferenças da prevalência dos indicadores entre os quintis de riqueza mais ricos e mais pobres foram calculadas de forma absoluta e relativa. Comparando os quatro indicadores de alimentação complementar, o IASSP teve o melhor desempenho, com 10 países, entre os 11 avaliados, apresentando prevalência acima de $80 \%$. $O$ indicador com pior desempenho foi o AMA, com apenas um país, entre os oito avaliados, com prevalências acima de 60\%. Na quase totalidade dos países, a população mais pobre possuía prevalências de indicadores de alimentação complementar menores que os mais ricos.

Palavras-chave Alimentação complementar, Determinantes sociais de saúde, Saúde pública, Política nutricional 


\section{Introdução}

A alimentação complementar pode ser compreendida como o processo iniciado quando o aleitamento materno exclusivo não é mais suficiente para atender às necessidades nutricionais da criança, sendo essencial a introdução de outros alimentos de forma complementar ao leite materno. Esse processo deve ser iniciado no sexto mês e se estende até os 24 meses de vida, devendo ser composto por alimentos em quantidade, frequência, variedade e consistência adequadas ${ }^{1}$. Práticas alimentares inadequadas para a criança nesse período estão associadas a desnutrição e obesidade, déficit de desenvolvimento e aumento da mortalidade infantil, principalmente entre as populações menos favorecidas ${ }^{2,3}$, podendo resultar, no longo prazo, em desempenho intelectual e capacidade de trabalho deficientes ${ }^{4,5}$.

Desigualdades sociais podem ser uma das causas do acesso insuficiente a alimentos seguros e de qualidade entre os mais pobres, e estão relacionadas a maiores níveis de insegurança alimentar doméstica, desnutrição e mortalidade infantil, sendo agravadas particularmente nas economias em desenvolvimento que passam por transição demográfica e nutricional $l^{6-8}$.

As hipóteses deste estudo são que os países possuem padrões de alimentação complementar diferentes entre si e que as desigualdades sociais determinam que a população mais pobre de cada país, quando comparada com a mais rica, possui menos acesso à alimentação complementar adequada e saudável. Este estudo tem como objetivo avaliar, nos países da América Latina e do Caribe, o papel das desigualdades sociais nos padrões de alimentação complementar entre países e intrapaíses.

\section{Métodos}

Trata-se de um estudo ecológico que utilizou dados de inquéritos de base populacional. Foram avaliadas duas bases de dados, uma oriunda de pesquisas nos moldes da Demographic and Health Surveys (DHS), outra nos moldes da Multiple Indicator Cluster Surveys (MICS). Os dados agregados foram obtidos no site do UNICEF".

A DHS e a MICS têm como sujeitos de pesquisa as mulheres em idade fértil ( 15 a 45 anos) e seus filhos até cinco anos de idade, adotando coleta de dados padronizados nos países em desenvolvimento por meio de questionários traduzidos para os idiomas locais, com periodicidade de aplicação variável. Os questionários abordam, entre outros temas, informações sobre aleitamento materno e alimentação complementar, permitindo comparações diretas entre as pesquisas, uma vez que o desenho do questionário e a qualidade da implementação são suficientemente semelhantes entre a DHS e MICS, entre países e ao longo do tempo.

Além disso, a DHS e a MICS utilizam estruturas de amostragem complexas em três estágios, estratificando por região e por áreas urbanas e rurais, utilizando os clusters censitários de cada país e tendo o domicílio como unidade de análise. Os agregados familiares de cada estrato são selecionados aleatoriamente a partir de listas de enumeração baseadas em informações de rastreio do censo. Os dados foram obtidos de ciclos da DHS e da MICS realizados entre 2008 e 2016.

A unidade de análise dessa pesquisa foram 16 países, sendo obtidas as prevalências de indicadores de alimentação complementar, divididos por quintis de riqueza, além de informações socioeconômicas e demográficas, tais como: população total e porcentagem de população urbana, renda per capita, Índice de Desenvolvimento Humano (IDH) e coeficiente de Gini. Nem sempre os indicadores de alimentação complementar estavam disponíveis para todos os países da amostra.

Com base nos indicadores de alimentação complementar preconizados pela $\mathrm{OMS}^{10}$, foram selecionados aqueles disponíveis nas bases de dados da UNICEF, a saber: "introdução alimentar de alimentos sólidos, semissólidos e pastosos" (IASSP), "diversidade alimentar mínima" (DAM), "frequência alimentar mínima" (FAM) e "alimentação mínima aceitável” (AMA).

O indicador IASSP foi calculado a partir da proporção de crianças de 6-8 meses de idade que receberam alimentos sólidos, semissólidos ou pastosos, dividida pelo total de crianças nessa faixa etária. Para o indicador DAM, foi calculada a proporção de crianças de 6-23 meses de idade que receberam alimentos de quatro ou mais grupos de alimentos entre os sete definidos pela OMS, dividida pelo total de crianças nessa faixa etária. Os sete grupos de alimentos para o cálculo da DAM foram: Grupo 1 - grãos, raízes e tubérculos; Grupo 2 - legumes e nozes; Grupo 3 - produtos lácteos (leite, iogurte, queijo); Grupo 4 - alimentos à base de carne (carne, peixe, aves e fígado/carne de órgãos); Grupo 5 - ovos; Grupo 6 - frutas e legumes ricos em vitamina A; Grupo 7 - outras frutas e produtos hortícolas.

$\mathrm{O}$ indicador FAM foi calculado a partir da proporção de crianças amamentadas e não ama- 
mentadas com 6-23 meses de idade que recebem alimentos sólidos, semissólidos e pastosos (mas também incluem alimentos lácteos para crianças não amamentadas). O mínimo é definido como: duas vezes para bebês amamentados com idade entre 6-8 meses; três vezes para crianças amamentadas com idade entre 9-23 meses; quatro vezes para crianças não amamentadas com idade entre 6-23 meses ${ }^{10}$.

$\mathrm{O}$ indicador AMA é um indicador composto, calculado a partir da proporção de crianças amamentadas com idade de 6-23 meses que atenderam aos critérios da diversidade alimentar mínima e da frequência mínima de refeições durante o dia anterior, dividida pelas crianças amamentadas de 6-23 meses, além da proporção de crianças não-amamentadas com idade de 6-23 meses que receberam pelo menos duas alimentações de leite e que atenderam aos critérios da diversidade alimentar mínima, não incluindo alimento lácteos, e a frequência mínima de refeições durante o dia anterior, dividida pelas crianças não amamentadas de 6-23 meses ${ }^{10}$.

A desigualdade social pode ser definida como um produto das relações sociais e econômicas entre grupos da sociedade, podendo ser medida por faixas de renda, em que são consideradas as médias dos mais ricos em comparação às dos mais pobres ${ }^{11-13}$. Dessa forma, além dos quintis de riqueza, consideramos. para a análise da desigualdade social, fatores como IDH, coeficiente de GINI e renda per capita.

Os quintis de riqueza foram obtidos por meio do índice de riqueza (Wealth Index, em inglês), e foram utilizados como variável de exposição, sendo o quintil mais pobre a referência. Esses quintis se baseiam em escores derivados de análises de componentes principais obtidos de uma lista de bens de consumo e de características das casas. O primeiro quintil (Q1) representa 20\% das famílias mais pobres, e o último quintil (Q5), $20 \%$ das famílias mais ricas.

$\mathrm{O}$ IDH se propõe a avaliar o bem-estar de uma população, especialmente das crianças, sendo uma medida síntese que considera as dimensões de riqueza, alfabetização, educação, esperança de vida, natalidade e outras. Os níveis de IDH variam de 0 a 1 (quanto mais próximo de 1 , maior o desenvolvimento humano). O coeficiente de GINI é uma medida resumo de desigualdade, podendo variar de 0 (igualdade perfeita) a 1 (desigualdade máxima) ${ }^{14}$. A renda per capita é um dos indicadores socioeconômicos que avaliam o grau de desenvolvimento econômico de determinado lugar, sendo uma média da divisão do produto nacional bruto pelo número total de habitantes ${ }^{15}$.

A correlação entre os indicadores socioeconômicos selecionados e os indicadores de alimentação complementar foram estimados por meio da correlação de Spearman, que visa determinar a força da relação entre as variáveis, que varia de -1 (correlação negativa perfeita) a +1 (correlação positiva perfeita), com intervalo de confiança de 95\%. Quando esse coeficiente é igual a 0 (zero), significa que não há correlação entre as variáveis. Quanto mais próximo aos valores extremos (-1 ou +1$)$, maior é a associação entre as variáveis ${ }^{16}$. Consideramos ausência de correlação valores $\leq$ 0,20 .

As prevalências dos indicadores IASSP, DAM, FAM e AMA foram tabuladas conforme o país e os quintis de riqueza. As diferenças das prevalências dos indicadores entre os quintis mais ricos e mais pobres foram calculadas de forma absoluta (subtraindo os valores extremos, prevalência entre os "mais ricos" menos a prevalência entre os "mais pobres") e de forma relativa (razão de prevalência entre os "mais ricos" e "mais pobres") 17 .

Por se tratar de análise de dados secundários, disponíveis publicamente, e sem identificadores pessoais, este estudo segue os preceitos éticos e está isento de avaliação por comitê de ética, conforme a Resolução no 510, de 7 de abril de 2016, da CONEP.

\section{Resultados}

Dos 24 países da América Latina e do Caribe acompanhados pela DHS e a MICS, apenas 16 dispunham de informações consistentes sobre alimentação complementar: Argentina (201112), Belize (2011), Bolívia (2008), Costa Rica (2011), El Salvador (2014), Guatemala (201415), Guiana (2014), Haiti (2012), Honduras (2011-12) Jamaica (2011), México (2015), Panamá (2013), Paraguai (2016), Peru (2012), República Dominicana (2014) e Suriname (2010).

Considerando os indicadores socioeconômicos dos países estudados, o percentual de população no ambiente urbano teve grande variação entre os países: a Argentina apresentou 91,1 \% da população residindo no meio urbano, enquanto na Guiana 28,5\% residiam nesse ambiente. Em relação ao IDH e ao Índice de GINI, os países apresentaram perfis homogêneos, sendo o Haiti o país com os piores resultados nos indicadores analisados. Outro indicador considerado foi a renda per capita, variando de US\$10.610 na Ar- 
gentina até US\$ 750 no Haiti (Tabela 1). No entanto, não foram encontradas correlações entre esses indicadores socioeconômicos e os indicadores de alimentação complementar nos países observados, uma vez que o valores do coeficiente de Spearman ficaram próximos de zero.

Em relação à introdução de alimentos sólidos, semissólidos e pastosos (IASSP), entre os 11 países com dados disponíveis, a prevalência variou de $79,8 \%$ na Guatemala a $96,6 \%$ na Argentina, uma diferença de 16,8 pontos percentuais. Comparando a população mais rica com a mais pobre de cada país, a diferença absoluta e relativa intrapaís na prevalência da IASSP foi maior na Guiana, seguida de Peru, El Salvador e Bolívia, respectivamente. Os países com menor diferença absoluta foram Haiti e Argentina, nessa ordem. Na Guiana, a introdução alimentar entre os mais ricos teve prevalência $61 \%$ maior em relação aos mais pobres, já na Argentina a introdução alimentar entre os mais ricos apresentou prevalência $2 \%$ menor em relação aos mais pobres (Tabela 2 ).

No que se refere ao indicador de diversidade alimentar mínima (DAM), nos 11 países analisados foi encontrada prevalência que variou de 25,4\% no Haiti até $82,2 \%$ no Peru, uma diferença de 56,8 pontos percentuais. Comparando a população mais rica em relação à população mais pobre, observa-se que a diferença absoluta intrapaíses na prevalência da DAM foi mais acentuada na Bolívia, na Guatemala e no Peru. Já Guiana, México e República Dominicana apresentaram menor diferença. Em relação à diferença relativa intrapaís para esse indicador, observa-se que foi maior no Haiti, na Bolívia e na Guatemala, e menor no México, na Guiana e em El Salvador. No Haiti a diversidade alimentar entre os mais ricos teve prevalência $90 \%$ maior em relação aos mais pobres, enquanto no México a prevalência de diversidade alimentar dos mais ricos foi $17 \%$ maior em comparação aos mais pobres (Tabela 3).

Quanto ao indicador de frequência alimentar mínima (FAM), nos 14 países com dados disponíveis, foi encontrada variação na prevalência de $42,0 \%$ na Jamaica a $86,9 \%$ em El Salvador, diferença de 44,9 pontos percentuais entre os países. Comparando a população mais rica em relação à população mais pobre, observa-se que a diferença absoluta intrapaís na prevalência da FAM foi maior no Haiti, na Guiana e no Panamá. Os países com menor diferença absoluta foram Honduras, El Salvador e República Dominicana. Já a diferença relativa intrapaís para FAM foi maior no Haiti, na Jamaica e no Panamá, e menor em Honduras, El Salvador e Guatemala. A prevalência da frequência alimentar mínima no Haiti foi $81 \%$ maior entre os ricos em relação aos mais pobres, já em Honduras a frequência alimentar mínima dos mais ricos foi apenas 5\% maior em comparação aos mais pobres (Tabela 4).

Tabela 1. Variáveis socioeconômicas e demográficas dos países analisados.

\begin{tabular}{|c|c|c|c|c|c|c|}
\hline País & Ano & População & $\begin{array}{l}\text { \% Pop. } \\
\text { urbana }\end{array}$ & IDH & Gini & $\begin{array}{l}\text { Renda per } \\
\text { capita }\end{array}$ \\
\hline Argentina & 2011 & 41.656 .879 & 91,1 & 0,822 & 42,3 & 10.610 \\
\hline Belize & 2015 & 359.288 & 44,0 & 0,706 & $53,1^{I}$ & 4.580 \\
\hline Bolívia & 2010 & 9.918 .242 & 66,4 & 0,649 & $46,1^{\mathrm{II}}$ & 1.810 \\
\hline Costa Rica & 2011 & 4.600 .474 & 72,9 & 0,758 & 48,7 & 8.060 \\
\hline El Salvador & 2014 & 6.281 .189 & 66,2 & 0,678 & 41,6 & 3.810 \\
\hline Guatemala & 2014 & 15.923 .559 & 51,1 & 0,637 & 48,3 & 3.450 \\
\hline Guiana & 2014 & 763.393 & 28,5 & 0,638 & $44,5^{\mathrm{III}}$ & 4.040 \\
\hline Haiti & 2012 & 10.289 .210 & 54,8 & 0,483 & 41,1 & 750 \\
\hline Honduras & 2011 & 8.505 .646 & 52,3 & 0,614 & 56,2 & 1.900 \\
\hline Jamaica & 2011 & 2.289 .493 & 53,9 & 0,725 & $45,4^{\mathrm{IV}}$ & 4.590 \\
\hline México & 2015 & 12.589 .0949 & 79,2 & 0,762 & $43,4^{\mathrm{V}}$ & 9.860 \\
\hline Panamá & 2013 & 3.838 .462 & 66,0 & 0,780 & 51,5 & 10.500 \\
\hline Paraguai & 2016 & 6.725 .308 & 75,5 & $0,693^{\mathrm{VI}}$ & 47,9 & 4.060 \\
\hline Peru & 2012 & 30.158 .966 & 77,6 & 0,731 & 44,7 & 5.670 \\
\hline República Dominicana & 2014 & 10.405 .844 & 78,1 & 0,718 & 44,1 & 6.090 \\
\hline Suriname & 2010 & 526.103 & 51,66 & 0,703 & $57,60^{\mathrm{VII}}$ & 8.303 \\
\hline
\end{tabular}

Fonte: Autores. 
Tabela 2. Introdução de alimentos sólidos, semissólidos e pastosos na alimentação de crianças de seis a oito meses por quintil de riqueza e país.

\begin{tabular}{|c|c|c|c|c|c|c|c|c|c|}
\hline \multirow{3}{*}{ País } & \multirow{3}{*}{$\begin{array}{c}\text { Fonte } \\
\text { Ano } \\
\end{array}$} & \multicolumn{4}{|c|}{ Quintil de riqueza $(\mathrm{WI})^{\mathrm{I}}$} & \multicolumn{2}{|c|}{ Total } & \multirow[b]{2}{*}{$\mathrm{DA}^{\mathrm{II}}$} & \multirow{3}{*}{$\mathrm{DR}^{\mathrm{II}}$} \\
\hline & & \multicolumn{2}{|c|}{ Quintil 1 } & \multicolumn{2}{|l|}{ Quintil 5} & \multirow[b]{2}{*}{ Prevalência } & \multirow[b]{2}{*}{$\mathbf{n}$} & & \\
\hline & & Prevalência & $\mathbf{n}$ & Prevalência & $\mathbf{n}$ & & & & \\
\hline \multirow[t]{2}{*}{ Argentina } & MICS & 97,7 & 35.808 & 96,0 & 22195 & 96,6 & 148004 & $-1,61$ & 0,98 \\
\hline & $2011 / 12$ & $(93,3-99,2)$ & & $(86,8-98,8)$ & & $(94,1-98,1)$ & & & \\
\hline \multirow[t]{2}{*}{ Bolívia } & DHS & 75,9 & 118 & 94,8 & 59 & 83,0 & 406 & 18,92 & 1,25 \\
\hline & 2008 & $(65,0-84,2)$ & & $(85,8-98,2)$ & & $(75,2-90,1)$ & & & \\
\hline \multirow[t]{2}{*}{ El Salvador } & MICS & 79,8 & 98 & 98,9 & 47 & 90,3 & 378 & 19,09 & 1,24 \\
\hline & 2014 & $(67,0-88,5)$ & & $(91,7-99,9)$ & & $(85,9-93,5)$ & & & \\
\hline \multirow[t]{2}{*}{ Guatemala } & DHS & 74,4 & 171 & 82,4 & 98 & 80,8 & 646 & 7,94 & 1,11 \\
\hline & $2014 / 15$ & $(64,8-82,2)$ & & $(71,1-89,9)$ & & $(76,5-84,3)$ & & & \\
\hline \multirow[t]{2}{*}{ Guiana } & MICS & 56,4 & 43 & 90,9 & 40 & 80,9 & 219 & 34,50 & 1,61 \\
\hline & 2014 & $(37,7-73,5)$ & & $(73,4-97,3)$ & & $(72,1-87,4)$ & & & \\
\hline \multirow[t]{2}{*}{ Haiti } & DHS & 86,4 & 76 & 93,1 & 27 & 85,3 & 259 & 6,70 & 1,07 \\
\hline & 2012 & $(80,1-92,8)$ & & $(88,5-97,3)$ & & $(81,3-89,8)$ & & & \\
\hline \multirow[t]{2}{*}{ Honduras } & DHS & 79,6 & 125 & 88,3 & 76 & 88,9 & 525 & 8,70 & 1,11 \\
\hline & $2011 / 12$ & $(71,3-86,0)$ & & $(74,4-95,1)$ & & $(84,4-92,4)$ & & & \\
\hline \multirow[t]{2}{*}{ México } & MICS & 86,4 & 93 & 92,7 & 35 & 82,3 & 369 & 6,33 & 1,07 \\
\hline & 2015 & $(71,7-94,1)$ & & $(75,5-98,1)$ & & $(65,8-91,9)$ & & & \\
\hline \multirow[t]{2}{*}{ Paraguai } & MICS & 79,4 & 53 & 92,1 & 30 & 87,1 & 207 & 12,72 & 1,16 \\
\hline & 2016 & $(54,1-92,6)$ & & $(75,8-97,7)$ & & $(79,1-92,4)$ & & & \\
\hline \multirow[t]{2}{*}{ Peru } & DHS & 73,7 & 70 & 98,5 & 66 & 87,2 & 415 & 24,80 & 1,33 \\
\hline & 2012 & $(64,3-79,8)$ & & $(92,6-99,7)$ & & $(81,6-90,3)$ & & & \\
\hline República & MICS & 78,7 & 359 & 85,0 & 153 & 80,5 & 1217 & 6,31 & 1,08 \\
\hline Dominicana & 2014 & $(72,1-84,0)$ & & $(76,0-91,0)$ & & $(77,0-83,7)$ & & & \\
\hline
\end{tabular}

${ }^{\mathrm{I}} \mathrm{WI}$ : wealth index. ${ }^{\mathrm{II}} \mathrm{DA}$ : diferença absoluta. ${ }^{\mathrm{III}} \mathrm{DR}$ : diferença relativa

Fonte: Autores.

Em relação ao indicador de alimentação mínima aceitável (AMA) foi observada, nos oito países analisados, prevalência que variou de 13,6\% no Haiti a $64,5 \%$ em El salvador, uma diferença de 50,9 pontos percentuais. Quando se compara a população mais rica com a mais pobre, a diferença absoluta intrapaís na prevalência da AMA foi maior na Guatemala, em El Salvador e Honduras. Os países com menor diferença absoluta foram Haiti, Guiana e Paraguai. Já a diferença relativa foi maior no Haiti, na Guatemala e na Guiana, e menor em El Salvador, México e Honduras. No Haiti, a alimentação mínima aceitável entre os mais ricos teve prevalência $141 \%$ maior em relação aos mais pobres, uma diferença menor foi observada em El Salvador, onde a alimentação mínima aceitável dos mais ricos foi 33\% maior em comporação aos mais pobres (Tabela 5).

Aa analisar os quatro indicadores de alimentação complementar, o IASSP teve o melhor desempenho, com 10 países, entre os 11 avaliados, apresentando prevalência acima de $80 \%$. Em seguida, o FAM teve desempenho regular, com 4 países, entre 14, com prevalência superior a $80 \%$. O indicador DAM teve desempenho intermediário, com 4 países, entre os 11 avaliados, apresentando prevalências superiores a $60 \%$. O indicador com pior desempenho foi o AMA, com apenas um país, entre os oito avaliados, com prevalências acima de $60 \%$.

\section{Discussão}

Os indicadores de alimentação mínima aceitável e diversidade alimentar mínima tiveram o pior desempenho entre os estudados, com baixas prevalências entre os países e grande disparidade dos mais ricos para os mais pobres de cada país, o que sugere violação do direito humano à alimentação adequada das crianças, uma vez que o Estado tem a obrigação de promover condições para garantir 


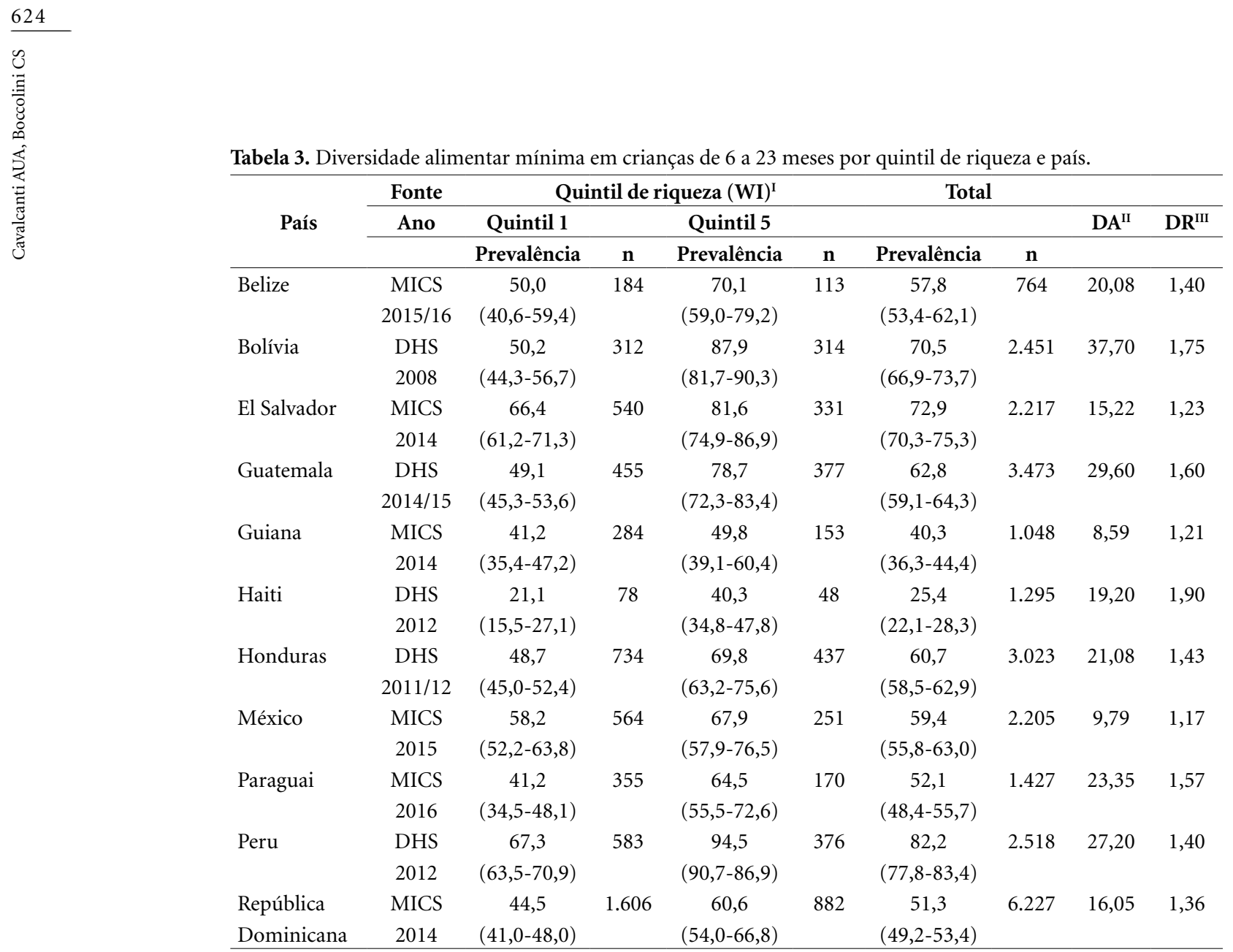

${ }^{\text {I}}$ WI: wealth index. ${ }^{\text {II } D A}$ : diferença absoluta. ${ }^{\text {III } D R: ~ d i f e r e n c ̧ a ~ r e l a t i v a . ~}$

Fonte: Autores.

o acesso e a utilização de alimentos e provê-los a indivíduos ou grupos incapazes de obtê-los por conta própria ${ }^{18}$. O indicador de diversidade alimentar pode ser mais sensível à não disponibilidade de alimento, à falta de acessibilidade e de estabilidade do acesso, o que consequentemente interfere no desempenho do indicador composto de alimentação mínima aceitável. O comportamento dos indicadores se assemelha ao observado em outros países que apresentaram o indicador de introdução alimentar e frequência com melhor desempenho em relação aos demais ${ }^{19,20}$.

O coeficiente de Spearman não conseguiu mostrar correlação entre alimentação complementar e as variáveis IDH, coeficiente de GINI e renda per capita, o que pode estar relacionado ao número reduzido de países considerados neste estudo. No entanto, foi observado que a população do quintil mais pobre dos países estudados teve menos acesso à alimentação complementar consistente e diversificada, quando comparada com a população do quintil mais rico, observan- do-se um gradiente socioeconômico entre essas classes. As exceções foram a Argentina, onde o quintil mais rico da população teve prevalência de introdução de alimentos sólidos, semissólidos e pastosos na idade recomendada menor que o quintil mais pobres, e o Haiti, que não apresentou variação entre o quintil mais rico em relação ao mais pobre para esse indicador. Esse gradiente de prevalência entre os níveis socioeconômicos também foi observado em países asiáticos e africanos, onde os mais pobres tinham piores indicadores do que os mais ricos ${ }^{19-22}$.

Vários estudos enfatizaram que os orçamentos familiares das populações mais pobres são insuficientes para obter uma alimentação diversificada. Mesmo quando grupos de baixa renda desenvolvem estratégias eficientes de compra, o orçamento alimentar pode não ser adequado para obter a alimentação recomendada ${ }^{23,24}$, indicando relação direta entre desigualdades sociais e alimentação ${ }^{23}$ onde subgrupos populacionais mais abastados não são apenas mais saudáveis 
Tabela 4. Frequência alimentar mínima de crianças de 6 a 23 meses por quintil de riqueza e país.

\begin{tabular}{|c|c|c|c|c|c|c|c|c|c|}
\hline \multirow{3}{*}{ País } & \multirow{2}{*}{$\begin{array}{c}\text { Fonte } \\
\text { Ano } \\
\end{array}$} & \multicolumn{4}{|c|}{ Quintil de riqueza $(\mathrm{WI})^{\mathrm{I}}$} & \multicolumn{2}{|c|}{ Total } & \multirow[b]{2}{*}{$\mathrm{DA}^{\mathrm{II}}$} & \multirow[b]{2}{*}{$\mathrm{DR}^{\mathrm{II}}$} \\
\hline & & Quintil 1 & & Quintil 5 & & & & & \\
\hline & & Prevalência & $\mathbf{n}$ & Prevalência & $\mathbf{n}$ & Prevalência & $\mathbf{n}$ & & \\
\hline \multirow[t]{2}{*}{ Argentina } & MICS & 58,6 & 250.672 & 75,1 & 149410 & 68,1 & 930.878 & 16,53 & 1,28 \\
\hline & $2011 / 12$ & $(52,4-64,6)$ & & $(66,5-82,2)$ & & $(64,9-71,1)$ & & & \\
\hline \multirow[t]{2}{*}{ Belize } & MICS & 60,8 & 160 & 79,7 & 87 & 67,6 & 613 & 18,90 & 1,31 \\
\hline & 2011 & $(51,8-69,0)$ & & $(67,4-88,1)$ & & $(62,8-72,0)$ & & & \\
\hline \multirow[t]{2}{*}{ Costa Rica } & MICS & 70,4 & 211 & 85,2 & 93 & 79,4 & 688 & 14,82 & 1,21 \\
\hline & 2011 & $(59,8-79,2)$ & & $(72,1-92,8)$ & & $(74,7-83,5)$ & & & \\
\hline \multirow[t]{2}{*}{ El Salvador } & MICS & 82,7 & 540 & 93,2 & 331 & 86,9 & 2.217 & 10,51 & 1,13 \\
\hline & 2014 & $(78,4-86,3)$ & & $(87,5-96,5)$ & & $(85,0-88,7)$ & & & \\
\hline \multirow[t]{2}{*}{ Guatemala } & DHS & 79,1 & 929 & 89,6 & 482 & 82,4 & 3.473 & 10,58 & 1,13 \\
\hline & $2014 / 15$ & $(76,0-81,8)$ & & $(85,3-92,8)$ & & $(80,9-83,9)$ & & & \\
\hline \multirow[t]{2}{*}{ Guiana } & MICS & 52,6 & 284 & 80,5 & 153 & 63,0 & 1.048 & 27,84 & 1,53 \\
\hline & 2014 & $(45,4-59,7)$ & & $(73,0-86,2)$ & & $(58,8-67,0)$ & & & \\
\hline \multirow[t]{2}{*}{ Haiti } & DHS & 35,4 & 456 & 64,2 & 226 & 44,2 & 1.295 & 28,79 & 1,81 \\
\hline & 2012 & $(30,7-40,4)$ & & $(55,8-71,8)$ & & $(41,0-47,4)$ & & & \\
\hline \multirow[t]{2}{*}{ Honduras } & DHS & 83,9 & 734 & 88,2 & 437 & 86,1 & 3.023 & 4,25 & 1,05 \\
\hline & $2011 / 12$ & $(81,0-86,4)$ & & $(83,2-91,8)$ & & $(84,5-87,5)$ & & & \\
\hline \multirow[t]{2}{*}{ Jamaica } & MICS & 34,5 & 118 & 52,6 & 78 & 42,0 & 482 & 18,13 & 1,53 \\
\hline & 2011 & $(25,2-45,2)$ & & $(38,3-66,6)$ & & $(36,2-48,1)$ & & & \\
\hline \multirow[t]{2}{*}{ México } & MICS & 70,5 & 564 & 86,4 & 251 & 81,3 & .2205 & 15,94 & 1,23 \\
\hline & 2015 & $(64,2-76,0)$ & & $(75,6-92,9)$ & & $(78,0-84,1)$ & & & \\
\hline \multirow[t]{2}{*}{ Panamá } & MICS & 49,8 & 34.518 & 75,7 & 8330 & 63,5 & 118.433 & 25,91 & 1,52 \\
\hline & 2013 & $(42,9-56,7)$ & & $(60,4-86,4)$ & & $(59,2-67,6)$ & & & \\
\hline \multirow[t]{2}{*}{ Paraguai } & MICS & 63,9 & 355 & 75,1 & 170 & 74,7 & 1.427 & 11,18 & 1,17 \\
\hline & 2016 & $(56,9-70,4)$ & & $(63,7-83,8)$ & & $(71,3-77,8)$ & & & \\
\hline República & MICS & 69,9 & 1.606 & 86,3 & 882 & 79,8 & 6.227 & 16,39 & 1,23 \\
\hline Dominicana & 2014 & $(66,9-72,8)$ & & $(81,5-90,0)$ & & $(78,2-81,2)$ & & & \\
\hline \multirow[t]{2}{*}{ Suriname } & MICS & 49,6 & 335 & 72,4 & 148 & 64,3 & 1.104 & 22,77 & 1,46 \\
\hline & 2010 & $(43,4-55,8)$ & & $(61,5-81,1)$ & & $(60,6-67,8)$ & & & \\
\hline
\end{tabular}

${ }^{\mathrm{I}}$ WI: wealth index. ${ }^{\mathrm{II}} \mathrm{DA}$ : diferença absoluta. ${ }^{\mathrm{III}} \mathrm{DR}$ : diferença relativa.

Fonte: Autores.

como têm acesso a dietas de melhor qualida$\mathrm{de}^{24,25}$. Outro ponto importante se relaciona ao acesso a alimentos que podem estar submetidos a relações climáticas, de produção e fatores conjunturais que levem a um quadro de vulnerabilidade alimentar, devido, por exemplo, a oscilações de mercado ${ }^{26}$.

A introdução de alimentos sólidos, semissólidos e pastosos (IASSP), apesar de ter sido o indicador com maior prevalência observada entre os países avaliados, possui baixa capacidade discriminativa, pois possui pequena diferença absoluta entre e intrapaíses. Além disso, esse indicador não afere a qualidade dos alimentos ofertados, uma vez que estudos mostram cada vez mais práticas inadequadas de introdução da alimentação complementar, com a oferta de alimentos não recomendados, como ultraprocessados e com alto teor de açúcar, bem como a interrupção do aleitamento materno ${ }^{27-29}$.

Além da introdução alimentar na idade recomendada pela OMS, as crianças precisam de um número mínimo de refeições e de diversidade dos alimentos. No entanto, muitos países em desenvolvimento, inclusive os que foram analisados neste estudo, ainda enfrentam o desafio de atender aos padrões mínimos de qualidade dietética para crianças ${ }^{30}$.

O segundo indicador com melhor desempenho foi o FAM, com uma diferença absoluta expressiva entre os países analisados, sendo a maior diferença relativa intrapaíses no Haiti. Esse indi- 
Tabela 5. Alimentação mínima aceitável de crianças de 6 a 23 meses por quintil de riqueza e país.

\begin{tabular}{|c|c|c|c|c|c|c|c|c|c|}
\hline \multirow{3}{*}{ País } & \multirow{3}{*}{$\begin{array}{c}\text { Fonte } \\
\text { Ano } \\
\end{array}$} & \multicolumn{4}{|c|}{ Quintil de riqueza $(\mathrm{WI})^{\mathrm{I}}$} & \multicolumn{2}{|c|}{ Total } & \multirow[b]{2}{*}{$\mathrm{DA}^{\mathrm{II}}$} & \multirow[b]{2}{*}{ DR $^{\text {II }}$} \\
\hline & & \multirow{2}{*}{$\begin{array}{c}\text { Quintil 1 } \\
\text { Prevalência }\end{array}$} & \multicolumn{3}{|c|}{ Quintil 5 } & \multirow[b]{2}{*}{ Prevalência } & \multirow[b]{2}{*}{$\mathbf{n}$} & & \\
\hline & & & $\mathbf{n}$ & Prevalência & $\mathbf{n}$ & & & & \\
\hline \multirow[t]{2}{*}{ El Salvador } & MICS & 57,5 & 540 & 76,8 & 331 & 64,5 & 2.217 & 19,3 & 1,33 \\
\hline & 2014 & $(52,3-62,5)$ & & $(68,9-83,2)$ & & $(61,7-67,2)$ & & & \\
\hline \multirow[t]{2}{*}{ Guatemala } & DHS & 42,6 & 929 & 65,0 & 482 & 52,1 & 3.473 & 22,4 & 1,52 \\
\hline & $2014 / 15$ & $(38,4-46,9)$ & & $(59,1-70,6)$ & & $(50,0-54,1)$ & & & \\
\hline \multirow[t]{2}{*}{ Guiana } & MICS & 26,9 & 284 & 40,8 & 153 & 27,8 & 1.048 & 13,9 & 1,51 \\
\hline & 2014 & $(21,8-32,8)$ & & $(31,2-51,2)$ & & $(24,3-31,5)$ & & & \\
\hline \multirow[t]{2}{*}{ Haiti } & DHS & 8,9 & 456 & 21,5 & 226 & 13,6 & 1.295 & 12,6 & 2,41 \\
\hline & 2012 & $(6,3-12,4)$ & & $(15,4-29,2)$ & & $(11,3-16,2)$ & & & \\
\hline \multirow[t]{2}{*}{ Honduras } & DHS & 43,9 & 734 & 62,8 & 437 & 54,8 & 3.023 & 18,9 & 1,43 \\
\hline & $2011 / 12$ & $(40,2-47,6)$ & & $(56,2-68,9)$ & & $(52,4-57,1)$ & & & \\
\hline \multirow[t]{2}{*}{ México } & MICS & 42,4 & 564 & 60,4 & 251 & 48,4 & 2.205 & 18,0 & 1,42 \\
\hline & 2015 & $(36,1-49,0)$ & & $(49,1-70,6)$ & & $(44,6-52,2)$ & & & \\
\hline \multirow[t]{2}{*}{ Paraguai } & MICS & 30,3 & 355 & 45,2 & 170 & 39,7 & 1.427 & 14,9 & 1,49 \\
\hline & 2016 & $(24,3-36,9)$ & & $(36,4-54,4)$ & & $(36,1-43,4)$ & & & \\
\hline República & MICS & 34,8 & 1.606 & 52,2 & 882 & 43,0 & 6.227 & 17,4 & 1,50 \\
\hline Dominicana & 2014 & $(31,5-38,3)$ & & $(47,1-57,3)$ & & $(41,2-44,9)$ & & & \\
\hline
\end{tabular}

IVI: wealth index. ${ }^{\text {II } D A: ~ d i f e r e n c ̧ a ~ a b s o l u t a . ~}{ }^{\mathrm{III}} \mathrm{DR}$ : diferença relativa.

Fonte: Autores.

cador está relacionado à frequência com que a criança recebe alimentos conforme preconizado pela OMS, o que nem sempre acontece, principalmente em famílias mais pobres, uma vez que a renda exerce influência direta na disponibilidade de alimento e na quantidade de refeições da família. No entanto, é importante considerar que prevalências mais altas nesse indicador podem ter ocorrido pela consideração de lanches na sua pontuação, definidos como alimentos consumidos entre as refeições, fáceis de preparar e que geralmente a criança come sozinha ${ }^{10}$.

Em estudos realizados nos países em desenvolvimento, a prevalência da frequência adequada das refeições também se mostrou mais elevada em relação a outros indicadores de alimentação complementar, tal como o indicador de diversidade alimentar mínima ${ }^{31,32}$.

$\mathrm{O}$ indicador diretamente relacionado à diversidade alimentar (DAM) apresentou resultados preocupantes, com baixa prevalência na maioria dos países, além de grande diferença absoluta entre os países, e distância elevada da prevalência observada entre os mais pobres e os mais ricos. Vale ressaltar que o indicador de diversidade alimentar é uma contagem de todos os grupos alimentares consumidos, incluindo alimentos ultraprocessados, não considerando as quantidades.
Em relação ao indicador DAM, um fato interessante a ser destacado diz respeito aos resultados apresentados pelo Haiti, que apresenta a prevalência mais baixa eentre os países analisados. Quando observados os quintis de riqueza dentro do país, observa-se menor variação entre os quintis mais pobre e o mais rico em relação aos demais países, indicando baixa diversidade alimentar em todos os quintis no Haiti, o que, segundo Ayoya e colaboradores ${ }^{33}$, pode ser atribuído ao impacto de desastres naturais e à pobreza.

$\mathrm{O}$ indicador AMA, referente a diversidade e frequência de alimentos, foi o que apresentou pior desempenho, e variação considerável, na diferença absoluta entre países, com valores acima de $50 \%$, indicando forte determinação do contexto em que as pessoas vivem na diversificação dos grupos alimentares consumidos. Por ser um indicador composto, o AMA implica que as crianças precisam receber tanto uma alimentação diversificada como um número recomendado de refeições, o que pode ser difícil em ambientes socioeconômicos mais pobres. No Haiti, existe uma discrepância intrapaís entre valores absolutos e relativos do AMA: observa-se a maior diferença relativa intrapaís $(\mathrm{RP}=2,41)$ e uma diferença absoluta mais discreta $(12,6 \%)$, o que pode ser explicado pela baixa prevalência em todos os quintis de riqueza, com apenas $13,6 \%$ das crian- 
ças recebendo uma alimentação mínima aceitável. O que faz sentido quando analisamos o fato de o Haiti apresentar um dos piores cenários de desnutrição e baixo peso em crianças na região da América Latina e do Caribe. A desnutrição infantil tem sido um grande problema de saúde pública no país: pesquisa populacional realizada em 2005 e 2006 revelou que duas em cada dez crianças menores de cinco anos de idade estavam abaixo do peso $^{34}$.

Pode-se concluir que os indicadores relacionados à qualidade e à diversidade da alimentação, DAM e AMA, evidenciam dois pontos importantes: primeiro, que existe associação direta entre maior poder aquisitivo e consumo mais diversificado de alimentos; e segundo, que o contexto (no caso, o país) em que a pessoa vive pode determinar a disponibilidade e a diversidade geral de alimentos, afetando coletivamente os mais ricos e os mais pobres, conforme observado na diferença absoluta intra e entre países.

Tais achados também foram observados por Issaka e colaboradores ${ }^{25}$, que, utilizando o índice de riqueza familiar como indicador indireto do status socioeconômico do agregado familiar, encontraram associação significativa entre a pobreza doméstica e a diversidade alimentar inadequada em Gana e na Nigéria. Outras associações negativas semelhantes entre a diversidade alimentar mínima e o baixo status socioeconômico também foram encontradas em estudos que utilizam dados da DHS realizados em países em desenvolvimento da Ásia e da África ${ }^{19,35,36}$.

Apesar das baixas prevalências de indicadores de qualidade e diversidade alimentar, existem soluções para melhorar esses indicadores: Haddad e colaboradores ${ }^{37}$ afirmam ser amplamente aceito que, quando as economias crescem e a pobreza diminui, a nutrição infantil melhora devido ao maior acesso a alimentos, à melhoria da assistência materno-infantil e a melhores serviços de saúde pública. A esse respeito, o estudo de Smith e Haddad ${ }^{38}$ mostrou evidências de que o crescimento econômico exerce influência positiva sobre a nutrição infantil.

No entanto, embora as taxas dos indicadores demonstrem variações entre os países, as práticas alimentares parecem sub-ótimas até mesmo nas famílias mais ricas. Assim, esses resultados explicitam o fato de que a capacidade familiar para comprar alimentos necessários é um pré -requisito para uma alimentação complementar satisfatória. Isso mostra a necessidade de uma compreensão mais abrangente sobre práticas alimentares, entendendo a segurança alimentar e nutricional em suas dimensões relacionadas ao acesso regular e constante a alimentos de qualidade, em quantidades suficientes, sem comprometer as outras necessidades básicas e tendo como base práticas alimentares promotoras de saúde.

Dessa forma, medidas de posição absoluta ou relativa são importantes, sendo particularmente relevantes ao considerar a pobreza, que pode ser definida em um sentido absoluto ao comparar determinada renda com um ponto de referência estático ou, em um sentido relativo, ao confrontar determinada renda com a distribuição geral de renda em uma população $0^{39,40}$.

Apesar do uso de dados nacionalmente representativos, o estudo apresentou algumas limitações. $\mathrm{O}$ indicador de introdução alimentar não avalia o momento da introdução de alimentos, considerando crianças que receberam alimentos antes dos seis meses. Além disso, o indicador DAM não contabilizou o leite materno como um grupo de alimento, uma vez que o indicador deve refletir apenas os alimentos complementares. Também é importante considerar que o índice socioeconômico, obtido por meio de uma escada de riqueza relativa, gerada a partir de componentes principais, apresenta limitações ao discriminar a realidade das famílias. Outra limitação do estudo são as diferenças nos anos de realização das pesquisas e dos indicadores socioeconômicos correlacionados. Apesar desses pontos, o estudo fornece evidências relevantes sobre a alimentação complementar na América Latina e no Caribe.

Assim, conclui-se que apenas uma pequena parcela das crianças estão se beneficiando de práticas de alimentação complementar, principalmente no quintil mais pobre dos países analisados, o que mostra a influência das desigualdades na alimentação infantil. São necessários esforços para melhorar a alimentação das crianças, para que sobrevivam, cresçam e se desenvolvam. Para garantir o direito à alimentação adequada e atender às Metas de Nutrição Global $2025^{41}$, a maioria dos países da América Latina e do Caribe precisa fortalecer seus esforços no combate à desnutrição e às desigualdades sociais, além de fortalecer os sistemas de informação de saúde para permitir o monitoramento dos indicadores nutricionais adequados e de forma continuada. Alcançar um crescimento saudável exige mais do que intervenções específicas na nutrição. Dessa forma, recomenda-se a intensificação das políticas públicas intersetoriais com foco na alimentação complementar como garantia do direito a uma alimentação saudável. 


\section{Colaboradores}

Os autores AUA Cavalcanti e CS Boccolini participaram da concepção, planejamento, análise, interpretação e redação do trabalho. Ambos também aprovaram a versão final a ser publicada.

\section{Referências}

1. World Health Organization (WHO). Complementary feeding: family foods for breastfeeding children [Internet]. Geneva: WHO; 2000. [acessado 2017 Maio 10]. Disponível em: http://whqlibdoc.who.int/hq/2000/ WHO_NHD_00.1.pdf

2. Black RE, Victora CG, Walker SP, Bhutta ZA, Christian P, Onis M, Ezzati M, Grantham-McGregor S, Katz J, Martorell R, Uauy R; Maternal and Child Nutrition Study Group. Maternal and child undernutrition and overweight in low-income and middle-income countries. The Lancet 2013; 382(9890):427-451.

3. Onis M, Branca F. Childhood stunting: a global perspective. Maternal \& Child Nutrition 2016; 12(Suppl. 1):12-26.

4. Bhutta ZA, Das JK, Rizvi A, Gaffey MF, Walker N, Horton S, Webb P, Lartey A, Black RE; Lancet Nutrition Interventions Review Group, the Maternal and Child Nutrition Study Group. Evidence-based interventions for improvement of maternal and child nutrition: what can be done and at what cost? The Lancet 2013; 382(9890):452-477.

5. Danaei G, Andrews KG, Sudfeld CR, Mccoy C, Peet E, Sania A, Fawzi WW. Risk factors for childhood stunting in 137 developing countries: a comparative risk assessment analysis at global, regional, and country levels. PLoS Medicine 2016; 13(11):e1002164.

6. Kien VD, Lee HY, Nam YS, Oh J, Giang KB, Minh HV. Trends in socioeconomic inequalities in child malnutrition in Vietnam: findings from the Multiple Indicator Cluster Surveys, 2000-2011. Global Health Action 2016; 9(1):29263.

7. Akombi BJ, Agho KE, Merom D, Renzaho AM, Hall JJ. Child malnutrition in sub-Saharan Africa: a meta -analysis of demographic and health surveys (20062016). PloS one 2017;12(5):e0177338

8. Akombi BJ, Agho KE, Renzaho AM, Hall JJ, Merom DR. Trends in socioeconomic inequalities in child undernutrition: evidence from Nigeria Demographic and Health Survey (2003-2013). PLoS One 2019; 14(2):e0211883.

9. UNICEF Global Databases. Infant and Young Child Feeding, 2018 [Internet]. [acessado 2018 Set 25] Disponível em: https://data.unicef.org/topic/nutrition/ infant-and-young-child-feeding/

10. World Health Organization (WHO). Indicators for assessing infant and young child feeding practices. Part I: definition. Geneva: WHO; 2008.

11. Marmot M. Social determinants of health inequalities. Lancet 2005; 365(9464):1099-1104.

12. Arcaya MC, Arcaya AL, Subramanian SV. Inequalities in health: definitions, concepts, and theories. Global Health Action 2015; 8:27106.

13. McCartney G, Bartley M, Dundas R, Katikireddi SV, Mitchell R, Popham F, Walsh D, Wami W. Theorising social class and its application to the study of health inequalities. SSM - Population Health 2019; 7:015-15.

14. Gastwirth JL. The estimation of the Lorenz curve and Gini index, Rev. Econ and Statl 1972; 63(3):306-316. 
15. World Bank Group. World Development Indicators Online (WDI Online). World Bank Group; 2006.

16. Hinkle DE, Wiersma W, Jurs SG. Applied statistics for the behavioral sciences. Boston: Houghton Mifflin; 2003.

17. World Health Organization (WHO). Handbook on health inequality monitoring with a special focus on low-and middle-income countries [Internet]. Geneva: WHO; 2013. [acessado 2018 Set 25]. Disponível em: https://www.who.int/gho/health_equity/handbook/ en/

18. Organização das Nações Unidas (ONU). Declaración Universal de los Derechos Humanos; 1948. [acessado 2018 Set 30]. Disponível em: http://www.un.org/es/ documents/udhr/

19. Areja A, Yohannes D, Yohannis M. Determinants of appropriate complementary feeding practice among mothers having children 6-23 months of age in rural Damot sore district, Southern Ethiopia; a community based cross sectional study. BMC Nutr 2017; 3:82.

20. Harvey CM, Newell ML, Padmadas SS. Socio-economic differentials in minimum dietary diversity among young children in South-East Asia: evidence from Demographic and Health Surveys. Public Health Nutr 2018; 21(16):3048-3057.

21. Issaka AI, Agho KE, Page AN, Burns PL, Stevens GJ, Dibley MJ. Determinants of suboptimal complementary feeding practices among children aged 6-23 months in four anglophone West African countries. Matern Child Nutr 2015; 11(Suppl. 1): 14-30.

22. Darmon N, Drewnowski A. Contribution of food prices and diet cost to socioeconomic disparities in diet quality and health: a systematic review and analysis. Nutr Rev 2015; 73(10):643-660.

23. Hirvonen K, Bai Y, Headey D, Masters WA. Affordability of the EAT-Lancet reference diet: a global analysis. Lancet Glob Health 2020; 8(1):e59-e66.

24. Turrell G, Kavanagh A, Subramanian SV. Area variation in mortality in Tasmania (Australia): the contributions of socioeconomic disadvantage, social capital and geographic remoteness. Health \& Place 2006. 12(3):291-305.

25. Gutiérrez-Camacho C, Méndez-Sánchez L, Klünder -Klünder M, Clark P, Denova-Gutiérrez E. Association between sociodemographic factors and dietary patterns in children under 24 months of age: a systematic review. Nutrients 2019; 11(9):2006.

26. Mayén AL, Marques-Vidal P, Paccaud F, Bovet P, Stringhini S. Socioeconomic determinants of dietary patterns in low- and middle-income countries: a systematic review. Am J Clin Nutr 2014; 100(6):15201531.

27. Green M, Hadihardjono DN, Pries AM, Izwardy D, Zehner E, Huffman SL. High proportions of children under 3 years of age consume commercially produced snack foods and sugar-sweetened beverages in Bandung City, Indonesia. Matern Child Nutr 2019; 15(Suppl. 4):e12764.
28. Pries AM, Huffman SL, Champeny M, Adhikary I, Benjamin M, Coly AN, Diop EHI, Mengkheang K, Sy NY, Dhungel S, Feeley A, Vitta B, Zehner E. Consumption of commercially produced snack foods and sugar -sweetened beverages during the complementary feeding period in four African and Asian urban contexts. Matern Child Nutr. 2017; 13(Suppl. 2):e12412.

29. Hadihardjono DN, Green M, Stormer A, Agustino, Izwardy D, Champeny M. Promotions of breastmilk substitutes, commercial complementary foods and commercial snack products commonly fed to young children are frequently found in points-of-sale in Bandung City, Indonesia. Matern Child Nutr 2019; 15(Suppl. 4):e12808.

30. Heidkamp RA, Ayoya MA, Teta IN, Stoltzfus RJ, Marhone JP. Complementary feeding practices and child growth outcomes in Haiti: an analysis of data from Demographic and Health Surveys. Matern Child Nutr 2015; 11(4):815-828.

31. Marriott B, White A, Hadden L, Davies JC, Wallingofrd JC. How well are infant and young child World Health Organization (WHO) feeding indicators associated with growth outcomes? An example from Cambodia. Matern Child Nutr 2010 6(4):358-373.

32. Jones AD, Ickes SB, Smith LE, Mbuya MN, Chasekwa B, Heidkamp RA, Menon P, Zongrone AA, Stoltzfus RJ. World Health Organization infant and young child feeding indicators and their associations with child anthropometry: a synthesis of recent findings. Matern Child Nutr 2014; 10(1):1-17.

33. Ayoya, MA, Ngnie-Teta I, Séraphin MN, Mamadoultaibou A, Boldon E, Saint-Fleur JE, Bernard S. Prevalence and Risk Factors of Anemia among Children 6-59 Months Old in Haiti. Anemia 2013. 2013:502968.

34. Cayemittes M, Placide MF, Mariko S, Barrere B, Severe B, Alexandre C. Enquete mortalite, morbidite, et utilization des services, EMMUS-IV, Haiti 2005-2006. Calverton: Ministere de la Santé Publique et de la Population; 2007. [acessado 2018 Set 9]. Disponível em: http://pdf.usaid.gov/pdf_docs/PNADI945.pdf

35. Solomon D, Aderaw Z, Tegegne T.K. Minimum dietary diversity and associated factors among children aged 6-23 months in Addis Ababa, Ethiopia. Int J Equity Health 2017; 16(1):181.

36. Baek Y, Chitekwe S. Sociodemographic factors associated with inadequate food group consumption and dietary diversity among infants and young children in Nepal. PloS One 2019; 14(3):e0213610.

37. . Haddad L, Alderman H, Appleton S, Song L, Yohannes Y. Reducing child malnutrition: how far does income growth take us? World Bank Econ Rev 2003; 17:107-131.

38. Smith LC, Haddad L. How potent is economic growth in reducing undernutrition? What are the pathways of impact? New cross-country evidence. Econ Dev Cult Change 2002; 51:55-76.

39. Sen A. Poverty and famines: an essay on entitlement and deprivation. Oxford: Oxford University Press; 1983. 
40. Citro CF, Michael RT. Measuring poverty: a new approach. Washington: National Academies Press; 1995.

41. United Nations (UN). General Assembly Resolution adopted by the General Assembly on 1 April 2016. 70/259. United Nations Decade of Action on Nutrition (2016-2025). [acessado 2020 Fev 18]. Disponível em: https://undocs.org/pdf?symbol=en/A/RES/70/259

Artigo apresentado em 24/06/2020

Aprovado em 07/01/2021

Versão final apresentada em 09/01/2021

Editores-chefes: Romeu Gomes, Antônio Augusto Moura da Silva 